\title{
Ofatumumab: a novel monoclonal anti-CD20 antibody
}

This article was published in the following Dove Press journal:

Pharmacogenomics and Personalized Medicine

10 May 2010

Number of times this article has been viewed

Thomas S Lin

GlaxoSmithKline Oncology R\&D,

Collegeville, PA, USA
Correspondence: Thomas S Lin GlaxoSmithKline Oncology R\&D, Collegeville, PA, USA

Email thomas.s.lin@gsk.com
Abstract: Ofatumumab, a novel humanized monoclonal anti-CD20 antibody, was recently approved by the FDA for the treatment of fludarabine and alemtuzumab refractory chronic lymphocytic leukemia (CLL). Ofatumumab effectively induces complement-dependent cytotoxicity (CDC) in vitro, and recent studies demonstrated that ofatumumab also effectively mediates antibody-dependent cellular cytotoxicity (ADCC). Pharmacokinetic studies indicated that increased exposure to the antibody correlated with improved clinical outcome in CLL. Thus, pharmacogenomics may be important in identifying which patients are more likely to respond to ofatumumab therapy, although such studies have not yet been performed. Patients with the high-affinity FCGR3a $158 \mathrm{~V} / \mathrm{V}$ polymorphism may be more likely to respond to therapy, if ADCC is the primary in vivo mechanism of action of ofatumumab. Patients with increased expression of the complement defense proteins CD55 and CD59 may be less likely to respond if ofatumumab works in vivo primarily via CDC. Patients with increased metabolism and clearance of ofatumumab may have lower exposure and be less likely to respond clinically. Thus, pharmacogenomics may determine the responsiveness of patients to ofatumumab therapy.

Keywords: monoclonal antibody, CD20, CLL, NHL, lymphoma

\section{Introduction}

The introduction of monoclonal antibodies such as the chimeric anti-CD20 antibody rituximab (Rituxan ${ }^{\circledR}$, Genentech; MabThera ${ }^{\circledR}$, Roche) and the chimeric anti-CD52 antibody alemtuzumab (Campath- $1 \mathrm{H}^{\circledR}$, Genzyme) has revolutionized the treatment of B-cell lymphoproliferative disorders such as B-cell non-Hodgkin's lymphoma (B-NHL) and chronic lymphocytic leukemia (CLL). ${ }^{1-4}$ Unlike the idiotype vaccine which underwent clinical trials in follicular lymphoma (FL), monoclonal antibodies are not specific for an individual patient's tumor. Instead, antibodies recognize antigens which are expressed on the tumor cells of most or all patients with a certain type of cancer. However, these antigens are not truly tumor-specific; for example, CD20 is expressed on normal B lymphocytes, and CD52 is expressed on a variety of hematopoietic cells. Consequently, the toxicity profile of a monoclonal antibody depends largely on its antigen's expression on normal tissues. Monoclonal antibodies kill tumor cells through several mechanisms: antibody-dependent cellular cytotoxicity (ADCC), complement-dependent cytotoxicity (CDC), and direct apoptosis. ${ }^{5-7}$ Monoclonal antibodies can be classified as type I or II, depending on their biological activity and mechanism of action in in vitro assays. Type I antibodies translocate CD20 into lipid rafts and are therefore more potent inducers of CDC, but are weak inducers of apoptosis. Lipid rafts are sphingolipid and cholesterol enriched-enriched mircodomains of the 
plasma membrane which function as organizing platforms for cell signaling and receptor trafficking. The ability of anti-CD20 antibodies to induce CDC correlated with their ability to translocate CD20 into lipid rafts, whereas induction of apoptosis by anti-CD20 antibodies was independent of CD20 segregation into lipid rafts. In contrast, type II antibodies do not induce lipid rafts and are thus poor inducers of $\mathrm{CDC}$, but more effectively induce direct apoptosis. Both type I and II antibodies effectively induce ADCC. However, the clinical activity of a monoclonal antibody may depend upon the affinity of an individual patient's Fc receptor for the Fc fragment of the antibody. ${ }^{5}$ Thus, the activity of an antibody in an individual patient may depend on the patient's genetic or biological profile.

The monoclonal antibody which has made the greatest clinical impact to date in hematologic malignancies is rituximab, which was initially approved for the treatment of FL more than a decade ago. ${ }^{1}$ Rituximab and the majority of second-generation anti-CD20 antibodies in clinical study are type I antibodies and redistribute CD20 into lipid rafts, although several type II anti-CD20 antibodies are in preclinical and clinical development. While rituximab was initially indicated as single-agent therapy for relapsed FL, subsequent studies demonstrated its activity in combination with cytotoxic chemotherapeutic agents such as alkylating agents (cyclophosphamide, bendamustine) and nucleoside analogs (fludarabine, pentotstatin) in FL, diffuse large B-NHL (DLBCL) and CLL. ${ }^{2,8-13}$ In fact, the primary clinical use of rituximab is in combination regimens, particularly in DLBCL and CLL. In CLL single-agent rituximab has had limited clinical impact, but rituximab has significantly changed the treatment of CLL, particularly in the upfront setting, due to combinations with fludarabine-based cytotoxic chemotherapy such as FCR. ${ }^{2,11,12}$ In contrast to FL, in which studies have demonstrated that rituximab works primarily via ADCC, ${ }^{5}$ rituximab's primary mechanism of action in CLL is undefined. ${ }^{6}$

In order to improve upon the biological and clinical activity of rituximab, especially in the single-agent setting, several humanized monoclonal anti-CD20 antibodies have been engineered to improve ADCC or CDC. These agents are in various stages of preclinical and clinical investigation in B-NHL and CLL. To improve ADCC, particularly in patients whose $\mathrm{Fc}$ receptors bind rituximab with low affinity, the Fc portions of several of these antibodies have been modified to improve binding to the $\mathrm{Fc}$ receptor. Individuals who are homozygous for valine at amino acid 158 of $\mathrm{Fc}$ gamma receptor (FCGR)3a (V/V) respond significantly better to rituximab than patients who are homozygous for phenylalanine $(\mathrm{F} / \mathrm{F})$ or who are heterozygous at amino acid 158 (V/F). ${ }^{5}$ Thus, one potential pathway to improve upon the activity of rituximab is to modify the $\mathrm{Fc}$ portion, in order to enhance binding to the $\mathrm{Fc}$ receptor of patients with lowaffinity FCGR3a polymorphisms.

\section{Ofatumumab}

This review will focus on the second-generation antiCD20 antibody which has undergone the furthest clinical development, ofatumumab (HuMax-CD20 ${ }^{\mathrm{TM}}$, Genmab; Arzerra $^{\mathrm{TM}}$, GSK). ${ }^{71-16}$ Ofatumumab was recently approved by the Food and Drug Administration (FDA) for the treatment of fludarabine and alemtuzumab refractory CLL, and the drug has been submitted for approval for the treatment of CALL in Europe. In addition, ongoing clinical studies are examining the safety and efficacy of ofatumumab as single-agent therapy for FL and in combination therapy for CLL, FL and DLBCL. Ofatumumab is a fully humanized, high-affinity monoclonal antibody whose epitope on CD20 is distinct from rituximab's target. ${ }^{14}$ The membrane proximal epitope reognized by ofatumumab encompasses both the small and large loops of CD20. In contrast, rituximab's binding site on CD20 is distal to ofatumumab's epitope and involves only the large loop. Preclinical studies indicated that ofatumumab has higher affinity for CD20 and activates CDC more effectively than rituximab. ${ }^{7}$

The initial form of ofatumumab, $2 \mathrm{~F} 2$, was generated by immunization of human immunoglobulin (Ig) transgenic mice with NS/0 cells transfected with human CD20. 2F2 induced redistribution of $\mathrm{CD} 20$ into detergent insoluble plasma membrane fractions, consistent with translocation into lipid rafts. ${ }^{7}$ Thus, like rituximab, ofatumumab is a type I antibody. Cytotoxicity assays using the ARH-77 lymphoma cell line indicated that rituximab was unable to induce cytotoxicity with unfractionated blood but mediated efficient cell killing when incubated with a purified mononuclear cell fraction containing natural killer (NK) cells, confirming that rituximab induced killing via ADCC. In contrast, $2 \mathrm{~F} 2$ was able to induce cell killing of ARH-77 cells when incubated with unfractionated blood. Furthermore, 2F2 was able to mediate cytotoxicity in the presence of plasma, and heat inactivation of plasma abolished this cell killing, suggesting that $2 \mathrm{~F} 2$ induced cytotoxicity via complement fixation. Studies in SU-DHL-4 and Daudi lymphoma cells, which express high levels of CD20 and are sensitive to CDC, indicated that both rituximab and $2 \mathrm{~F} 2$ were able to induce lysis of these cell lines. However, 2F2 was more effective than rituximab at 
inducing $\mathrm{CDC}$ at low antibody concentrations. Furthermore, only $2 \mathrm{~F} 2$ was able to induce CDC of Raji lymphoma cells, which have moderate CD20 expression but high levels of the complement defense molecules CD55 and CD59. Thus, 2F2 induced CDC more effectively than rituximab. Studies with primary CLL cells suggested that this difference may be clinically significant in CLL. 2F2, but not rituximab, induced killing of CLL cells in the presence of unfractionated blood, whereas both antibodies induced only low levels of ADCC in the presence of mononuclear cells. ${ }^{7}$

Subsequent studies using C1q-depleted serum supplemented with low concentrations of $\mathrm{C} 1 \mathrm{q}$, which initiates the classical pathway of complement, further illustrated the enhanced ability of ofatumumab, or $2 \mathrm{~F} 2$, to induce CDC. ${ }^{17}$ These studies, which were conducted in Raji and Daudi lymphoma cell lines and primary CLL tumor cells, demonstrated that $\mathrm{C} 1 \mathrm{q}$ bound more avidly to ofatumumab-opsonized cells than to rituximab-opsonized cells, resulting in high levels of complement fixation and $\mathrm{CDC}$. Even when similar $\mathrm{C} 1 \mathrm{q}$ binding was obtained with higher levels of $\mathrm{C} 1 \mathrm{q}$, rituximabopsonized cells induced less complement fixation and CDC than ofatumumab-opsonized cells. Thus, the superior ability of ofatumumab to induce $\mathrm{CDC}$ resulted from both increased $\mathrm{C} 1 \mathrm{q}$ binding and enhanced ability to activate complement after initial binding of $\mathrm{C} 1 \mathrm{q} .{ }^{17}$

While these studies demonstrated that ofatumumab effectively induces $\mathrm{CDC}$, a recent abstract presented at the 2009 Annual Meeting of the American Society of Hematology (ASH) indicated that ofatumumab is also a potent inducer of ADCC. Craigen et al compared the ability of ofatumumab and rituximab to induce ADCC by purified NK cells from healthy donors expressing $\mathrm{V} / \mathrm{V}(\mathrm{n}=10)$ or $\mathrm{F} / \mathrm{F}(\mathrm{n}=10)$ at amino acid 158 of FCGR3a. ${ }^{18} \mathrm{Fc}$-mediated antibody binding to NK cells and ADCC were examined in a blinded study which incubated ARH-77 lymphoma cells with different concentrations of ofatumumab or rituximab in the presence of purified NK cells at an effector-to-target ratio of $5: 1$. Antibody binding was measured by flow cytometry in competition binding assays using ofatumumab or rituximab. Monomeric ofatumumab bound 4.4 times more strongly to NK cells expressing FCGR3a $158 \mathrm{~V} / \mathrm{V}$ than to cells expressing $158 \mathrm{~F} / \mathrm{F}$, with $\mathrm{EC}_{50} 900$ and $3970 \mathrm{mg} / \mathrm{mL}$, respectively $(P<0.0001)$. A similar 4.2 -fold difference in NK cell binding between FCGR3a $158 \mathrm{~V} / \mathrm{V}$ and $158 \mathrm{~F} / \mathrm{F}$ was found for rituximab $\left(\mathrm{EC}_{50} 1370\right.$ and $5720 \mathrm{mg} / \mathrm{mL}$, respectively, $P<0.0001)$. Of note, ofatumumab bound 1.5 -fold more tightly to both FCGR3a allotypes than rituximab $(P<0.0001)$. Ofatumumab induced potent NK-mediated
ADCC with cells from donors with FCGR3a $158 \mathrm{~V} / \mathrm{V}$ and $158 \mathrm{~F} / \mathrm{F}$, with $\mathrm{EC}_{50} 6.4$ and $17.6 \mathrm{ng} / \mathrm{mL}$, respectively $(P<0.0001)$. Higher concentrations of rituximab were required to induce $\mathrm{ADCC}$ by $\mathrm{NK}$ cells in this assay, with $\mathrm{EC}_{50} 12$ and $31 \mathrm{ng} / \mathrm{mL}$, respectively, for donors with FCGR3a $158 \mathrm{~V} / \mathrm{V}$ and $158 \mathrm{~F} / \mathrm{F}$. This 1.8-fold increase in ADCC potency between ofatumumab and rituximab $(P<0.0001)$ translated into increased killing of cells by ofatumumab, compared to rituximab, in the ADCC assay using NK cells from donors with FCGR3a $158 \mathrm{~V} / \mathrm{V}$ (39.7\% vs 27.6\%) and $158 \mathrm{~F} / \mathrm{F}(40.1 \%$ vs $35.4 \%) .{ }^{18}$ These studies indicated that ofatumumab effectively induces both ADCC and CDC. ${ }^{7,18}$

Binding studies using Fab' fragments indicated that 2F2 bound more tightly to CD20 than rituximab, and additional studies in DOHH cells demonstrated a slower off-rate for $2 \mathrm{~F} 2$ compared to rituximab. ${ }^{7}$ After 3 hours, more than $70 \%$ of $2 \mathrm{~F} 2$ remained bound to cells, compared to $30 \%$ for rituximab. The faster off-rate of rituximab resulted in antibody dissociation and a concomitant loss in CDC assays, compared to $2 \mathrm{~F} 2$. Cross-linking with sheep anti-human $\kappa$ antibody prevented dissociation of rituximab from the cell surface and improved the CDC activity of rituximab, confirming that the faster off-rate of rituximab resulted in inferior CDC. ${ }^{7}$ Epitope mapping, using both mutagenesis studies and a novel epitope analysis method using overlapping 15-mer peptides of the extracellular loops of CD20 and a Pepscan-based ELISA assay, demonstrated that rituximab bound to the large extracellular loop of CD20. In contrast, 2F2 recognized a distinct epitope N-terminal of rituximab's binding site; furthermore, the epitope of 2F2 included both the small and large loops of the extracellular domain of CD20. ${ }^{14}$

Thus, ofatumumab's preclinical profile suggests several potential mechanisms of advantage over rituximab (Table 1). In in vitro assays ofatumumab induced both $\mathrm{CDC}$ and $\mathrm{ADCC}$ more effectively than than did rituximab. ${ }^{7,18}$ While a higher concentration of ofatumumab was needed to bind NK cells from donors with the low affinity FCGR3a polymorphism $158 \mathrm{~F} / \mathrm{F}$ than from donors with the high affinity polymorphism $158 \mathrm{~V} / \mathrm{V}$, resulting in a higher concentration for induction of ADCC in

Table I Preclinical characteristics of ofatumumab

Ofatumumab is a type I antibody (lipid rafts)

Epitope on CD20 is distinct from rituximab's epitope

Epitope encompasses both the small and large loops of CD20

Ofatumumab binds CD20 more tightly with slower off-rate

Ofatumumab induces complement-dependent cytotoxicity (CDC)

Ofatumumab induces antibody-dependent cytotoxicity (ADCC)

Ofatumumab caused prolonged B-cell depletion in animal studies 
the low affinity patients, ofatumumab was more effective at binding NK cells and inducing ADCC than rituximab in both patient groups. ${ }^{18}$ In addition, ofatumumab recognized a distinct epitope on CD20 which is N-terminal to rituximab's epitope and encompasses both the small and large loops. ${ }^{14}$ Finally, ofatumumab bound more tightly, with a slower off-rate, than rituximab. ${ }^{7}$ Therefore, phase I/II clinical studies were initiated to determine whether these biological differences would translate into clinical activity in lymphoid malignancies.

\section{Clinical development in CLL}

A phase I/II study of oftatumumab was conducted in CLL, based on these in vitro studies as well as animal studies demonstating prolonged B-cell depletion in cynolmogus monkeys exposed to ofatumumab, compared to rituximab. Ofatumumab was given by intravenous (IV) infusion weekly for 4 doses to 33 patients with relapsed CLL. ${ }^{16}$ Median age of patients was 61 years (range 27-82), and 58\% were male. Four patients had Rai stage III/IV disease at screening, whereas 28 patients were stage I/II. Four patients had bulky lymphadenopathy greater than $5 \mathrm{~cm}$ in diameter. Patients were a median of 6.3 years (range 1.2-14) from diagnosis and had received a median of 3 prior therapies (range 1-9). Cohort 1 administered ofatumumab $100 \mathrm{mg}$ followed by 3 doses of $500 \mathrm{mg}$ to 3 patients, whereas 3 patients in cohort 2 received $300 \mathrm{mg}$ followed by 3 doses of $1000 \mathrm{mg}$. Cohort 3 administered $500 \mathrm{mg}$ followed by 3 doses of $2000 \mathrm{mg}$ to 27 patients. Twenty-seven patients experienced 246 adverse events (AEs), of which 150 were judged to be related to ofatumumab and 19 were grade 3 or 4 . Fifty-six percent of AEs were infusion-related and decreased in incidence and severity with subsequent infusions of ofatumumab. Two patients developed grade 4 neutropenia, and 2 patients experienced grade 3 thrombocytopenia judged to be related to therapy. Seventeen patients $(51 \%)$ experienced a total of 25 infections, most of which were grade 1-2. Three patients developed grade 3 infections (herpes zoster, nasopharyngitis, pneumonia), and one patient died of infectious interstitial pneumonitis. In total, 10 serious adverse events (SAEs) were observed in 9 patients, and the maximum tolerated dose (MTD) was not reached. One patient in cohort 3 was withdrawn from therapy due to transient grade 3 hepatitis after the first $500 \mathrm{mg}$ infusion of ofatumumab, but the other 32 patients were evaluable for response. Thirteen patients in cohort 3 responded (48\%), including one nodular partial response (nPR) and 12 PR (Table 2). Pharmacokinetic (PK) analysis demonstrated that clinical response to ofatumumab correlated with the drug's minimum or trough serum concentration $\left(\mathrm{C}_{\min }, P=0.009\right)$, maximum or peak concentration $\left(\mathrm{C}_{\max }, P=0.003\right)$, area under the curve (AUC, $P=0.006)$, and half-life $\left(\mathrm{t}_{1 / 2}, P=0.009\right)$.

Based on these promising results, a pivotal phase II licensing trial was initiated in patients with relapsed or refractory CLL. ${ }^{19}$ Patients were eligible for this study if they were refractory to both fludarabine and alemtuzumab (FA-REF) or were refractory to fludarabine and had bulky nodal disease defined as at least one lymph node of $5 \mathrm{~cm}$ or greater in dimension (bulky fludarabine refractory, BFR). The primary endpoint was overall response (OR) rate, and a planned interim analysis was performed when 66 patients were enrolled in the FA-REF group. At the time of the interim analysis, 154 patients had been enrolled, including 59 patients in the FA-REF group, 79 patients in the BFR group, and 16 patients who did not meet the eligibility criteria of either treatment cohort (Table 2). Median age of the 59 FA-REF patients was 64 years (range 41-86), 75\% were male, and 54\% had Rai stage III/IV disease. Median number of prior therapies was 5 (range 1-14), 93\% of patients had received alkylator therapy, and $59 \%$ had received rituximab therapy. Median age of the 79 BFR patients was 62 years (range 43-84), 72\% were male, and $70 \%$ had Rai stage III/IV disease. Median number

Table 2 Clinical studies of weekly single-agent ofatumumab

\begin{tabular}{|c|c|c|c|c|c|}
\hline Phase & $\mathbf{N}$ & Disease & Dosing schedule & Response rate & Median PFS \\
\hline$\overline{\mathrm{I} / \mathrm{II}}$ & $27^{\mathrm{a}}$ & CLL (relapsed) & $\begin{array}{l}500 \mathrm{mg} \text { week I } \\
2000 \mathrm{mg} \text { weeks 2-4 }\end{array}$ & $48 \%$ & Not reported \\
\hline II & $\begin{array}{l}59 \\
79\end{array}$ & $\begin{array}{l}\text { CLL (FA-REF) } \\
\text { CLL (BFR) }\end{array}$ & $\begin{array}{l}300 \mathrm{mg} \text { week I } \\
2000 \mathrm{mg} \text { weeks } 2-8 \\
2000 \mathrm{mg} \text { monthly } \times 4\end{array}$ & $\begin{array}{l}58 \% \\
47 \%\end{array}$ & $\begin{array}{l}5.7 \text { months } \\
5.9 \text { months }\end{array}$ \\
\hline $\mathrm{I} / \mathrm{II}$ & $38^{\mathrm{b}}$ & FL (relapsed) & $300-1000 \mathrm{mg}$ weeks $\mathrm{I}-4$ & $43 \%$ & 8.8 months \\
\hline II & 116 & FL (refractory) & $\begin{array}{l}300 \mathrm{mg} \text { week I } \\
500-1000 \mathrm{mg} \text { weeks } 2-8\end{array}$ & $11 \%$ & 6.0 months \\
\hline
\end{tabular}

aPatients in cohort 3; ${ }^{\mathrm{b}}$ Patients who were evaluable for response.

Abbreviations: $\mathrm{N}$, number of patients; PFS, progression-free survival; CLL, chronic lymphocytic leukemia; FA-REF, fludarabine and alemtuzumab refractory; BFR, bulky fludarabine refractory; FL, follicular lymphoma. 
of prior therapies was 4 (range 1-16), 92\% of patients had received alkylator therapy, and 54\% had received rituximab therapy. Ninety-three percent of FA-REF patients and 100\% of BFR patients had at least one lymph node of $5 \mathrm{~cm}$ or greater in dimension by physical examination and/or radiographic imaging.

Ofatumumab was given weekly for 8 doses, followed by 4 maintenance monthly doses. ${ }^{19}$ All patients received $300 \mathrm{mg}$ for the first infusion, and a dose of $2000 \mathrm{mg}$ was administered for each of the remaining 11 infusions; thus, patients received a total of 22,300 mg. Patients received acetaminophen $1000 \mathrm{mg}$ and cetirizine $10 \mathrm{mg}$ or equivalent prior to infusions. Patients also received prednisolone $100 \mathrm{mg}$ or equivalent prior to infusions 1,2 and 9; the glucocorticoid dose could be reduced for other infusions if the first two infusions were well-tolerated. Antibiotic prophylaxis was not mandated.

Infusion-related reactions, nearly all of which were grade 1-2 and occurred primarily during the first two infusions, were seen in $64 \%$ of FA-REF patients and $61 \%$ of BFR patients. ${ }^{19}$ The most common AEs were infections $(67 \%)$, cough $(18 \%)$, diarrhea (16\%), anemia (16\%), fatigue $(15 \%)$, fever $(15 \%)$ and neutropenia (15\%). AEs judged to be related to ofatumumab treatment included 2 cases each of grade 3-4 thrombocytopenia and grade 3-4 hemolytic anemia and 1 case of grade 3 febrile neutropenia. Ninety-two patients experienced a total of 189 infections, of which 139 (74\%) were grade 1-2. Among 37 grade 3-4 infections, pneumonia $(n=14)$ and other respiratory tract infections $(n=6)$ were most common. Thirteen infections resulted in death, including sepsis $(n=6)$, pneumonia $(\mathrm{n}=5)$, fusarium infection $(\mathrm{n}=1)$ and progressive multifocal leukoencephalopathy (PML, $\mathrm{n}=1$ ).

Response was assessed by an Independent Review Committee (IRC) using the 1996 NCI-WG response criteria. The OR rate was $58 \%$ and $47 \%$ for FA-REF and BFR patients, respectively, with stable disease in $31 \%$ and $41 \%$ of FA-REF and BFR patients, respectively. All responders achieved PR except for one patient who attained a complete response (CR). The OR rate among patients who previously received a rituximab-containing regimen was $54 \%$ and $44 \%$ in the FA-REF and BFR groups, respectively. ${ }^{19}$ Ofatumumb was rapidly effective in responding patients, with approximately $80 \%$ of responses occurring within 2 months of treatment initiation. The median duration of response was 7.1 months in FA-REF patients and 5.6 months in BFR patients. Median progression-free survival (PFS) was 5.7 months and 5.9 months for FA-REF and BFR patients, respectively. Median overall survival (OS) was 13.7 months and 15.4 months for FA-REF and BFR patients, respectively. An exploratory landmark analysis was performed at week 12 to determine if there was a correlation between response and OS. Median OS was significantly longer among responding patients; the median OS had not yet been reached for responders in either the FA-REF or BFR groups, compared with 9.8 months and 10.2 months, respectively, for nonresponders. Based on the results of the planned interim analysis of this pivotal phase II study, ofatumumab was recently approved by the FDA for the treatment of fludarabine and alemtuzumab refractory CLL.

Osterborg et al examined the relationship among serum ofatumumab concentrations, baseline patient characteristics and clinical outcomes in this study, which he presented at the 2009 ASH meeting. ${ }^{20}$ Multivariate analysis demonstrated that a higher $\mathrm{C}_{\max }$ following the first dose of ofatumumab was associated with a lower percentage of bone marrow infiltration by CLL, lower Rai stage, lower lymphocyte count, small body surface area and lower total bilirubin. Notably, the first three associations were with parameters of lower tumor burden and, thus, slower antibody clearance. The likelihood of response correlated with three parameters of antibody exposure at dose 8, which was the final dose of weekly treatment. Responders demonstrated a 37\% higher $\mathrm{AUC}$, a $23 \%$ higher $\mathrm{C}_{\max }$ and a $91 \%$ higher $\mathrm{C}_{\min }$ at dose 8 than non-responders. Interestingly, no association was found between PK parameters at dose 12 and clinical response. In addition, higher $\mathrm{AUC}, \mathrm{C}_{\max }$ and $\mathrm{C}_{\min }$, as well as lower clearance, correlated with improved PFS at both dose 8 and dose 12 . However, exploratory multivariate analysis did not indicate that PK parameters independently predicted either ORR or PFS. Nonetheless, these studies indicated that increased exposure to ofatumumab may result in improved clinical outcome, although more detailed studies will need to be performed to define this relationship.

Wierda et al recently presented preliminary results of a randomized phase II study examining two doses of ofatumumab, $500 \mathrm{mg}$ and $1000 \mathrm{mg}$, in combination with standard dose fludarabine and cyclophosphamide (FC) every 28 days in previously untreated CLL. ${ }^{21}$ The median age of patients was 56 years (range 38-73), $46 \%$ of patients had Rai stage III/IV disease, and the median $\beta$-2-microglobulin level was $4.0 \mathrm{mg} / \mathrm{L}$. Patients received $300 \mathrm{mg}$ of ofatumumab IV on day 1 of cycle 1 , followed by fludarabine $25 \mathrm{mg} / \mathrm{m}^{2} \mathrm{IV}$ and cyclophosphamide $250 \mathrm{mg} / \mathrm{m}^{2} \mathrm{IV}$ on days 2 to 4 of cycle 1 . The ofatumumab dose was increased to $500 \mathrm{mg}(\mathrm{n}=31)$ or $1000 \mathrm{mg}(\mathrm{n}=30)$ on day 1 of cycles 2 to 6 , and FC was administered at the same doses on days 1 to 3 of cycles 2 to 6 . OR 
and $\mathrm{CR}$ rates were $75 \%$ and $41 \%$, and there was a trend toward a higher CR rate in the $1000 \mathrm{mg}$ arm (50\% vs 31\%). A higher $\mathrm{CR}$ rate was observed in patients with a $\beta$-2-microglobulin level $<4.0 \mathrm{mg} / \mathrm{L}$ compared to those with $\beta$-2-microglobulin level $\geq 4.0 \mathrm{mg} / \mathrm{L}$ ( $53 \%$ vs $29 \%$ ), and patients who completed all 6 planned cycles of therapy had higher OR (92\% vs 48\%) and CR (55\% vs $17 \%)$ rates than patients who received fewer than 6 cycles. While 4 patients came off study due to failure to respond, most patients withdrew from therapy due to toxicity, most notably cytopenias. Only 1 of 8 patients with del (17p13), corresponding to loss of p53, achieved CR. Due to short median follow-up time, no PFS data are available. The small sample size, high $\beta$-2-microglobulin level in both cohorts, and high percentage of $\operatorname{del}(17 \mathrm{p} 13)$ patients in the $1000 \mathrm{mg}$ treatment arm make it difficult to compare these results with historical results of other chemoimmunotherapy regimens such as FCR.

In summary, ofatumumab demonstrated significant single-agent activity in fludarabine and alemtuzumab refractory CLL and recently was approved by the FDA for this indication. ${ }^{19}$ Preliminary PK studies indicated that increased antibody exposure may correlate with improved clinical outcome, although no pharmacokinetic parameter independently predicted for OR rate or PFS. ${ }^{20} \mathrm{~A}$ small phase II study of ofatumuamb in combination with FC demonstrated activity, although comparison to historical results with rituximabbased combination regimens was limited by the small size of the study, high $\beta$-2-microglobulin level, and high percentage of patients with the poor-risk del(17p13) cytogenetic abnormality. ${ }^{21}$

\section{Ofatumumab in indolent B-NHL}

Ofatumumab has also demonstrated clinical activity in relapsed FL. A multi-center phase I/II study administered ofatumumab to 40 patients with relapsed FL. ${ }^{15}$ Median age was 58.5 years (range $34-75$ ), and $50 \%$ of patients were male. Median time from diagnosis was 4.5 years (range 0.7-17.1), and 31 patients were stage III/IV at screening. Patients had received a median of 2 prior therapies (range $1-8)$, and 15 patients had received prior rituximab either as monotherapy or in combination with cytotoxic chemotherapy. Ofatumumab was given at a dose of 300, 500, 700 or $1000 \mathrm{mg}$ IV weekly to 10 patients per cohort. Forty patients developed a total of 274 AEs, of which 190 were judged to be related to therapy. Similar to the experience in CLL, infusion toxicity was seen with the first ofatumumab dose in virtually all patients but extinguished with subsequent infusions. Ninety-five percent of AEs were grade 1 or 2 , but 7 patients developed a total of 8 grade 3 AEs judged to be related to therapy. Thirteen patients experienced a total of 20 infections; 18 infections were grade 1 or 2 and included 11 cases of upper respiratory tract infection. Two grade 3 infections (urinary tract infection, neutropenic sepsis) were reported as SAEs but were judged unrelated to ofatumumab. There was no relationship between dose and toxicity, and the MTD was not reached. Sixteen of 38 evaluable patients (43\%) responded (Table 2); 5 patients achieved a $\mathrm{CR}$, and 2 attained an unconfirmed $\mathrm{CR}$ $(\mathrm{CRu})$. The OR rate varied between $20 \%$ and $63 \%$ across cohorts, and there was no dose-response relationship. Nine of 14 evaluable patients who previously received rituximab responded (64\%), including 3 of 4 rituximab-refractory patients. Six of 10 patients in cohort 4 (1000 mg dose), 6 of 10 patients responded, including 4 of 5 patients who had failed prior rituximab therapy. Median time to progression (TTP) was 8.8 months for all subjects and 32.6 months for responding patients, and median duration of response was 29.9 months. Thus, ofatumumab demonstrated significant activity in FL patients who had relapsed after prior rituximab therapy. Ofatumumab caused immediate, profound B-cell depletion lasting 6 to 10 months after end of therapy, and $65 \%$ of evaluable patients converted from Bcl-2 positive to Bcl-2 negative peripheral blood. Clinical response did not correlate with $\mathrm{C}_{\max }$ or $\mathrm{AUC}$, but $\mathrm{t}_{1 / 2}$ and clearance correlated with clinical response at week 26 but not week 19 .

To determine if single agent ofatumumab has activity in patients with refractory FL, a multi-center study administered 8 weekly doses of ofatumumab to 116 patients with rituximab refractory FL. ${ }^{22}$ Patients were initially randomized to receive $500 \mathrm{mg}$ or $1000 \mathrm{mg}$ of ofatumumab. However, due to slow accrual, the study was amended to close the $500 \mathrm{mg}$ treatment arm. Median age of all patients was 61 years (range 37-82), 86\% of patients were Ann Arbor stage III/IV, and $75 \%$ of patients had intermediate or high-risk disease by the Follicular Lymphoma International Prognostic Index (FLIPI) including 47\% with high-risk disease. Patients were refractory to rituximab monotherapy (23\%), rituximab-based combination chemotherapy (38\%), or rituximab maintenance therapy following cytotoxic chemotherapy (39\%). Interestingly, $65 \%$ of patients were refractory to cytotoxic chemotherapy. Patients in the $500 \mathrm{mg}$ and $1000 \mathrm{mg}$ treatment cohorts had similar characteristics, with no significant differences between the two groups. All patients received $300 \mathrm{mg}$ for the first infusion, followed by $500 \mathrm{mg}(\mathrm{n}=30)$ or $1000 \mathrm{mg}$ $(\mathrm{n}=86)$ for their 7 remaining weekly infusions. Patients received acetaminophen $1000 \mathrm{mg}$ and cetirizine $10 \mathrm{mg}$ or equivalent prior to infusions, as well as prednisolone $100 \mathrm{mg}$ 
or equivalent prior to infusions 1 and 2; the glucocorticoid dose could be reduced for other infusions if the first two infusions were well-tolerated. Antibiotic prophylaxis was not mandated. Treatment was well tolerated, with primarily grade 1-2 infusion toxicity associated with the first ofatumumab dose which extinguished with subsequent infusions. There were only three cases of grade 3-4 non-hematologic, non-infectious toxicity ( 2 cough, 1 urticaria). Twenty-eight patients experienced infection, mostly grade 1-2 upper respiratory infections, with only 2 cases of grade 3 infection. Fifteen percent of patients experienced transient grade 3-4 neutropenia which resolved a few weeks after completion of therapy.

The primary endpoint of the study was OR rate (Table 2). The OR rate was $11 \%$, including $10 \%$ of patients in the $1000 \mathrm{mg}$ arm and $13 \%$ of patients who received $500 \mathrm{mg} .{ }^{22}$ Three patients attained $\mathrm{CR}$ or $\mathrm{CRu}$, and 10 patients achieved PR. Since there was no difference in primary endpoint between the two treatment arms, patients in both cohorts were combined for all other analyses. Patients who were refractory to rituximab monotherapy appeared to be most sensitive to single-agent ofatumumab; $22 \%$ of patients refractory to rituximab monotherapy responded, compared to $7 \%$ of patients refractory to rituximab conbination chemotherapy and $9 \%$ of patients refractory to rituximab maintenance therapy. Although the OR rate was $11 \%$, nearly half of patients experienced some diminution in the sum of the products of the diameters (SPD) of their target lesions. Median PFS was 6.0 months. Thus, ofatumumab demonstrated limited single-agent activity in this heavily pretreated, refractory population. Thus, current efforts in FL are focused on the use of ofatumumab monotherapy in patients with previously untreated FL or relapsed, rituximab-sensitive FL, as well as combination regimens in both rituximab-sensitive and rituximab-refractory FL.

\section{Role of pharmacogenomics}

What is the potential role of pharmacogenomics in ofatumumab therapy? The answer to this depends on the relative clinical importance of ADCC and CDC as potential mechanisms of action, as well as the clearance or metabolism of ofatumumab by patients' bodies (Table 3 ). The importance of polymorphisms at amino acid 158 of FCGR3a has been well demonstrated in FL patients treated with rituximab, which is thought to work primarily by ADCC in FL. ${ }^{5}$ Individuals with $158 \mathrm{~V} / \mathrm{V}$ respond significantly better to single-agent rituximab than patients with $158 \mathrm{~F} / \mathrm{F}$ or $158 \mathrm{~V} / \mathrm{F}$. However, a similar relationship between FCGR3a polymorphisms
Table 3 Potential role of pharmacogenomics in ofatumumab

Antibody-dependent cellular cytotoxicity (ADCC)
FCGR3a polymorphisms
I58 V/V (high affinity)
I58 F/F (low affinity)
I58 V/F (intermediate affinity)
Complement-dependent cytotoxicity (CDC)
CD55 and CD59
High expression (resistant to CDC)
Low expression (sensitive to CDC)
Exposure to drug-drug clearance
Increased metabolism of antibody (lower exposure/activity)
Increased antibody clearance (lower exposure/activity)

and response to therapy has not been reported for combination regimens of rituximab and cytotoxic chemotherapy in FL. Furthermore, the importance of FCGR3a polymorphisms in determining response to therapy has not been described for more aggressive B-NHL histologies such as DLBCL, and a study in CLL demonstrated no difference in clinical response to rituximab monotherapy between patients with $158 \mathrm{~V} / \mathrm{V}$ and those with $158 \mathrm{~F} / \mathrm{F}$ or $158 \mathrm{~V} / \mathrm{F}$. It is unclear if ofatumumab also is more effective in FL patients with the high-affinity $158 \mathrm{~V} / \mathrm{V}$ polymorphism, but recent data presented at the 2009 ASH meeting indicated that ofatumumab may work in vivo via ADCC. ${ }^{18}$ Therefore, studies to examine the effect of FCGR3a polymorphisms upon the response to ofatumumab, both as single-agent therapy and in combination regimens with chemotherapy, are ongoing. Similarly, pharmacogenomics may be important in determining patients' susceptibility to ofatumumab if $\mathrm{CDC}$ is the antibody's primary in vivo mechanism of action. In vitro preclinical studies demonstrated that ofatumumab, but not rituximab, was able to induce CDC of Raji lymphoma cells with high levels of the complement defense molecules CD55 and CD59. ${ }^{7}$ Thus, patients with high levels of CD55 and CD59 may potentially be more resistant to ofatumumab therapy than patients with lower levels of these complement inhibitory proteins. Interestingly, a recent report indicated that the complement regulatory proteins CD46 and CD59 were higher in 15 patients with B-NHL who responded to rituximab and chemotherapy than in 7 patients who failed to respond to chemotherapy. ${ }^{23}$ While this finding appears counterintutive, it should be noted that CDC is not thought to be an important mechanism of action of rituximab in FL and other B-NHL. Given the increased ability of ofatumumab to induce $\mathrm{CDC}$ in vitro, it would be intriguing to examine the effect of CD55 and CD59 expression upon the clinical response of FL patients to ofatumumab, in order to determine whether patients with increased expression of 
these complement inhibitory proteins are less likely to respond to ofatumumab based therapy.

Finally, the presentation by Osterborg et al at the 2009 ASH meeting demonstrated that increased exposure to ofatumumab correlated with clinical outcome in refractory CLL patients treated with the antibody. ${ }^{20}$ Lower tumor burden correlated with increased $\mathrm{C}_{\max }$, which along with increased AUC and $\mathrm{C}_{\min }$, correlated with an improved OR rate. Thus, pharmacogenomic differences which result in slower metabolism of ofatumumab, decreased antibody clearance, and increased exposure to ofatumumab may result in increased clinical activity of the antibody.

In summary, ofatumumab is a humanized monoclonal anti-CD20 antibody which was recently approved by the FDA for the treatment of fludarabine and alemtuzumab refractory CLL, and which is currently undergoing further clinical studies as monotherapy and in combination therapy in CLL and B-NHL such as FL. Ofatumumab effectively induced $\mathrm{CDC}$ in vitro, and recent studies demonstrated that ofatumumab is also an effective mediator of ADCC. PK studies indicated that increased exposure to the antibody correlated with improved clinical outcome including OR rate and PFS. Thus, pharmacogenomics may be important in identifying which patients are more likely to respond to ofatumumab therapy, although such studies have not yet been performed. Patients with the high-affinity FCGR3a $158 \mathrm{~V} / \mathrm{V}$ polymorphism may be more likely to respond to therapy, if ADCC is an important in vivo mechanism of action of ofatumumab. Patients with increased expression of the complement defense proteins CD55 and CD59 may be less likely to respond to ofatumumab if the antibody works in vivo primarily via CDC. Finally, patients with increased metabolism and clearance of ofatumumab may experience lower in vivo exposure to the antibody and be less likely to respond clinically. Thus, several potential avenues of pharmacogenomic research may help to delineate patients who are more or less susceptible to ofatumumab therapy.

\section{Disclosure}

The author is an employee of GSK.

\section{References}

1. McLaughlin P, Grillo-Lopez AJ, Link BK, et al. Rituximab chimeric antiCD20 monoclonal antibody therapy for relapsed indolent lymphoma: half of patients respond to a four-dose treatment program. J Clin Oncol. 1998;16:2825-2833.

2. Keating MJ, O'Brien S, Albitar M, et al. Early results of a chemoimmunotherapy regimen of fludarabine, cyclophosphamide, and rituximab as initial therapy for chronic lymphocytic leukemia. J Clin Oncol. $2005 ; 23: 4079-4088$.
3. Coiffier B, Lepage E, Briere J, et al. CHOP chemotherapy plus rituximab compared with $\mathrm{CHOP}$ alone in elderly patients with diffuse large B-cell lymphoma. N Engl J Med. 2002;346:235-242.

4. Keating MJ, Flinn I, Jain V, et al. Therapeutic role of alemtuzumab (Campath-1H) in patients who have failed fludarabine: results of a large international study. Blood. 2002;99:3554-3561

5. Cartron G, Dacheux L, Salles G, et al. Therapeutic activity of humanized anti-CD20 monoclonal antibody and polymorphism in IgG Fc receptor Fc gamma RIIIa gene. Blood. 2002;99:754-758.

6. Farag SS, Flinn IW, Modali R, Lehman TA, Young D, Byrd JC. Fc gamma RIIIa and Fe gamma RIIa polymorphisms do not predict response to rituximab in B-cell chronic lymphocytic leukemia. Blood. 2004;103:1472-1474.

7. Teeling JL, French RR, Cragg MS, et al. Characterization of new human CD20 monoclonal antibodies with potent cytolytic activity against non-Hodgkin lymphomas. Blood. 2004;104:1793-1800.

8. Marcus R, Imrie K, Belch A, et al. CVP chemotherapy plus rituximab compared with CVP as first-line treatment for advanced follicular lymphoma. Blood. 2005;105:1417-1423.

9. van Oers MH, Klasa R, Marcus RE, et al. Rituximab maintenance improves clinical outcome of relapsed/resistant follicular non-Hodgkin lymphoma in patients both with and without rituximab during induction: results of a prospective randomized phase 3 intergroup trial. Blood. 2006;108:3295-3301.

10. Coiffier B, Lepage E, Briere J, et al. CHOP chemotherapy plus rituximab compared with $\mathrm{CHOP}$ alone in elderly patients with diffuse large B-cell lymphoma. $N$ Engl J Med. 2002;346:235-242.

11. Kay NE, Geyer SM, Call TG, et al. Combination chemoimmunotherapy with pentostatin, cyclophosphamide, and rituximab shows significant clinical activity with low accompanying toxicity in previously untreated B chroinc lymphocytic leukemia. Blood. 2007;109:405-411.

12. Byrd JC, Peterson BL, Morrison VA, et al. Randomized phase 2 study of fludarabine with concurrent versus sequential treatment with rituximab in symptomatic, untreated patients with B-cell chronic lymphocytic leukemia: results from Cancer and Leukemia Group B 9712 (CALGB 9712). Blood. 2003;101:6-14

13. Rummel MJ, Al-Batran SE, Kim SZ, et al. Bendamustine plus rituximab is effective and has a favorable toxicity profile in the treatment of mantle cell and low-grade non-Hodgkin's lymphoma. J Clin Oncol. 2005;23:3383-3389.

14. Teeling JL, Mackus WJ, Wiegman LJ, et al. The biological activity of human CD20 monoclonal antibodies is linked to unique epitopes on CD20. J Immunol. 2006;177:362-371.

15. Hagenbeek A, Gadeberg O, Johnson P, et al. First clinical use of ofatumumab, a novel fully human anti-CD20 monoclonal antibody in relapsed or refractory follicular lymphoma: Results of a phase $1 / 2$ trial. Blood. 2008;111:5486-5495.

16. Coiffier B, Lepretre S, Pedersen LM, et al. Safety and efficacy of ofatumumab, a fully humanized monoclonal anti-CD20 antibody, in patients with relapsed or refractory B-cell chronic lymphocytic leukemia: A phase 1-2 study. Blood. 2008;111:1094-1100.

17. Pawluczkowycz AW, Beurskens FJ, Beum PV, et al. Binding of submaximal $\mathrm{C} 1 \mathrm{q}$ promotes complement-dependent cytotoxicity (CDC) of B cells opsonized with anti-CD20 mAbs ofatumumab (OFA) or rituximab (RTX): considerably higher levels of CDC are induced by OFA than by RTX. J Immunol. 2009;183:749-758.

18. Craigen JL, Mackus WJ, Engleberts P, et al. Ofatumumab, a human Mab targeting a membrane-proximal small-loop epitope on CD20, induces potent NK cell-medited ADCC. [abstract]. Blood. 2009;114:687.

19. Wierda WG, Kipps TJ, Mayer J, et al. Ofatumumab as single-agent CD20 immunotherapy in fludarabine-refractory chronic lymphocytic leukemia. J Clin Oncol. 2010 Mar 1. [Epub ahead of print].

20. Osterborg A, Biilmann Ronn B, Jewell RC, et al. Correlation between serum ofatumumab concentrations, baseline patient characteristics and clinical outcomes in patients with fludarabine-refractory chronic lymphocytic leukemia (CLL) treated with single-agent ofatumumab. [abstract]. Blood. 2009;114:1334. 
21. Wierda WG, Kipps TJ, Durig J, et al. Ofatumumab combined with fludarabine and cyclophosphamide (O-FC) shows high activity in patients with previously untreated chronic lymphocytic leukemia (CLL): Results from a randomized, multicenter, international, two-dose, parallel group, phase II trial. [abstract] Blood. 2009;114:90-91.

22. Hagenbeek A, Fayad L, Delwail V, et al. Evaluation of ofatumumab, a novel human CD20 monoclonal antibody, as single agent therapy in rituximab-refractory follicular lymphoma. [abstract]. Blood. 2009; 114:385-386.
23. Dzietczenia J, Wrobel T, Mazur G, Poreba R, Jazwiec B, Kuliczkowski K. Expression of complement regulatory proteins: CD46, CD55, and CD59 and response to rituximab in patients with CD20(+) non-Hodgkin's lymphoma. Med Oncol. 2009. Aug 7 [Epub ahead of print].

\section{Publish your work in this journal}

Pharmacogenomics and Personalized Medicine is an international, peerreviewed, open access journal characterizing the influence of genotype on pharmacology leading to the development of personalized treatment programs and individualized drug selection for improved safety, efficacy and sustainability. This journal is indexed on the American Chemical
Society's Chemical Abstracts Service (CAS). The manuscript management system is completely online and includes a very quick and fair peer-review system, which is all easy to use. Visit http://www.dovepress. $\mathrm{com} /$ testimonials.php to read real quotes from published authors.

Submit your manuscript here: http://www.dovepress.com/pharmacogenomics-and-personalized-medicine-journal 